\title{
Autumn conference
}

\author{
Chang-Ju Kim (ib) https://orcid.org/0000-0003-4749-5795
}

The Autumn Conference of the Korean Society of Exercise Rehabilitation will be held at Gachon University Gil Hospital on November 2 (Saturday), 2019 under the theme of "Exercise Rehabilitation and the 4th Industrial Revolution."

Beginning with the theme presentation of "The exercise rehabilitation and the Fourth Industrial Revolution" by professor Khae-Hawn Kim of Gil Hospital, Gachon University, and invited lecture of doctor Sang-Won Lim titled "What training is required for aging of the fascia, cognitive ability?" is ready. The research presentations of professor Yun-Hee Sung titled "Understanding vestibular rehabilitation" and of professor Ji-Youn Kim titled "Understanding the maximization of utilization of sports facilities for the disabled by applying universal design technology: In the development of facilities, supplies, and operational guidelines" are prepared. We want to reveal the existence of exercise rehabilitation in the Fourth Industrial Revolution through academic conferences. Now is the time for a lot of discussion.

The first industrial revolution represented by steam engines and mechanization, which began in the United Kingdom in 1784, the second industrial revolution with full-scale production using electricity in 1870, and the third industrial revolution led by the computer informatization and automated production system led by the Internet in 1969. Next, it refers to the industrial change that is expected to build a virtual physics system that can control things automatically and intelligently by integrating real and virtual through robots or artificial intelligence (AI). The 4th Industrial Revolution is a next-generation industrial revolution in which innovative information is transformed by integrating cutting-edge information and communication technologies such as AI, the Internet of Things, big data, and mobile throughout the economy and society. Because of its characteristics, it has a greater impact on a wider scope and at a higher velocity than the existing industrial revolution.

In this development of the Fourth Industrial Revolution, it is necessary to discuss how to show the existence of exercise rehabilitation. The conference will discuss this seriously.

\section{CONFLICT OF INTEREST}

No potential conflict of interest relevant to this article was reported.

President of the Korean Society of Exercise Rehabilitation Department of Physiology, College of Medicine, Kyung Hee University, 26 Kyungheedae-ro, Dongdaemun-gu, Seoul 02447, Korea E-mail: changju@khu.ac.kr 